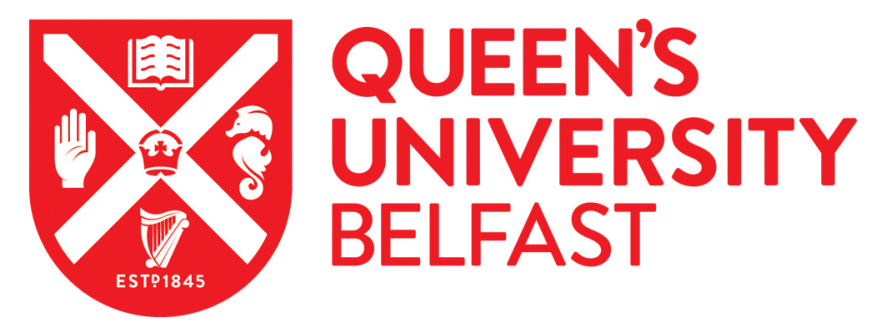

\title{
The road to 6G: Ten physical layer challenges for communications engineers
}

Matthaiou, M., Yurduseven, O., Ngo, H-Q., Morales-Jimenez, D., Cotton, S., \& Fusco, V. (2021). The road to 6G: Ten physical layer challenges for communications engineers. IEEE Communications Magazine, 59(1). https://doi.org/10.1109/MCOM.001.2000208

\section{Published in:}

IEEE Communications Magazine

\section{Document Version:}

Peer reviewed version

\section{Queen's University Belfast - Research Portal:}

Link to publication record in Queen's University Belfast Research Portal

\section{Publisher rights}

Copyright 2020 IEEE. This work is made available online in accordance with the publisher's policies. Please refer to any applicable terms of use of the publisher.

\section{General rights}

Copyright for the publications made accessible via the Queen's University Belfast Research Portal is retained by the author(s) and / or other copyright owners and it is a condition of accessing these publications that users recognise and abide by the legal requirements associated with these rights.

Take down policy

The Research Portal is Queen's institutional repository that provides access to Queen's research output. Every effort has been made to ensure that content in the Research Portal does not infringe any person's rights, or applicable UK laws. If you discover content in the Research Portal that you believe breaches copyright or violates any law, please contact openaccess@qub.ac.uk. 


\title{
The Road to 6G: Ten Physical Layer Challenges for Communications Engineers
}

\author{
Michail Matthaiou, Okan Yurduseven, Hien Quoc Ngo, David Morales-Jimenez, \\ Simon L. Cotton, and Vincent F. Fusco
}

\begin{abstract}
While the deployment of $5 \mathrm{G}$ cellular systems will continue well in to the next decade, much interest is already being generated towards technologies that will underlie its successor, 6G. Undeniably, 5G will have transformative impact on the way we live and communicate, yet, it is still far away from supporting the Internet-ofEverything (IoE), where upwards of a million devices per $\mathbf{k m}^{3}$ (both terrestrial and aerial) will require ubiquitous, reliable, low-latency connectivity. This article looks at some of the fundamental problems that pertain to key physical layer enablers for 6G. This includes highlighting challenges related to intelligent reflecting surfaces, cell-free massive MIMO and THz communications. Our analysis covers theoretical modeling challenges, hardware implementation issues and scalability among others. The article concludes by delineating the critical role of signal processing in the new era for wireless communications.
\end{abstract}

\section{INTRODUCTION}

Each generation of cellular networks has an average timescale of 10 years from original conception to full commercialization. Now, in the eve a new decade, $5 \mathrm{G}$ is becoming a commercial reality while all mobile operators and vendors around the world are competing for market primacy. 5G was originally envisioned to be a technological shift compared to $4 \mathrm{G}$ in terms of aggregate spectral and energy efficiency, as well as, latency and reliability [1]. After nearly eight years of intensive academic research and industrial testing, the lessons we have taken are the following: a) 5G can indeed support emerging data-hungry applications (e.g. ultra-fast broadband, high-definition video streaming), mainly through advances in the massive MIMO (MaMi) space; b) $5 \mathrm{G}$ is still falling short of supporting the so-called Internet-of-Everything (IoE), where myriads of devices in a geographic cube require either low-latency, ultra-reliable connectivity or wireless Gpbs Internet access by availing of the mm-

The authors are with the Institute of Electronics, Communications and Information Technology (ECIT), Queen's University, Belfast, Belfast BT3 9DT, U.K. (e-mail: m.matthaiou@qub.ac.uk).
wave/THz spectrum [2]. Having said the above, a number of experts are asking the grand questions: "what comes after 5G?" and "are we approaching the limits of wireless communications?". The answers to these questions are not straightforward, especially taking into account the ever increasing number of connected devices for the years to come. In this context, the idea of $6 \mathrm{G}$ has tentatively started to circulate within the wireless community, with the first overview articles appearing in the literature (see for example [2], [3] and references therein). The common ground of these narrative articles is that $6 \mathrm{G}$ will try to address the shortcomings of $5 \mathrm{G}$ by boldly (a) pushing the communication to higher frequency bands (mm-wave and $\mathrm{THz}$ ), (b) creating smart radio environments through reconfigurable surfaces and (c) by removing the conventional cell structures (aka cell-free massive MIMO (CF MaMi)). Unfortunately, transforming these speculative academic concepts into commercially viable solutions is a very challenging exercise. In this article, we identify ten of the most important challenges that need to be addressed in at the physical layer to boost followup research in the $6 \mathrm{G}$ ecosystem. Our discussion covers a broad spectrum of topics, from fundamental electromagnetic (EM) and signal processing (SP) topics to transceiver design and hardware implementations.

\section{IntELligEnt REFLECTING SURFACES (IRS)}

A reflective surface is a planar aperture synthesized using an array of sub-wavelength elements (or unit cells). Such a surface can be used to modulate the incoming waves into a desired wavefront upon reflection. Due to their sub-wavelength unit cell sampling, reflective surfaces can be considered a distinct form of metasurfaces, synthesized using an array of metamaterial unit cell elements. There has been a substantial amount of research conducted in EM wave control using metasurface apertures 
with applications ranging from imaging to EM invisibility [4]. Despite the fact that these structures are well understood within the applied EM community, their adoption in wireless communication networks has been under investigated to date. With the recent implementation of the 5G technology and the upcoming $6 \mathrm{G}$ networks, reflective surfaces offer the potential to substantially reduce the cost and complexity of the hardware layer while improving the total energy efficiency.

Wireless systems conventionally rely on a mature antenna technology to establish a communication link, which is known as phased arrays [5]. A phased array consists of individual antennas with dedicated phased shifting circuits and power amplifiers to synthesize the desired aperture wavefront. Phased arrays can be power hungry and exhibit a rather complex hardware architecture. Different from the phased array technology, metasurfaces do not rely on phase shifting circuits to modulate the phase of the incoming waves. Instead, metasurfaces rely on a holographic principle to achieve the desired phase modulation. The incoming wave illuminating the aperture surface acts as a reference-wave, which is converted to a desired wavefront upon reflection from the reflective metasurface aperture. Hence, the reflective surface can be thought of as the product of the interaction between the incident reference-wave and the desired reflection wavefront. Their major advantage is that they can synthesize any arbitrary waveform using this simple, yet strong, holographic principle without the need for expensive and power hungry phase shifters. Designing a reflective surface for wireless systems exhibits two main challenges:

Challenge 1: Unit cell phase range and phase quantization levels

An important limitation in the design process of a reflective surface is the achievable phase range of the unit cells synthesizing the aperture. Ideally, each unit cell across the reflective surface should provide a full phase control across a phase range of $0-2 \pi$. However, achieving this full-phase range may require a rather complex unit cell topology, with multiple layers complicating the hardware architecture. Another important constraint in the design process of a reflective surface is the quantization of the phase range of the unit cells. Even if a full phase range of $0-2 \pi$ is achieved, the number of quantization levels used to discretize this phase range has a direct impact on the fidelity of the synthe- sized wavefront upon reflection from the reflective surface. To investigate the effect of these design constraints, we consider a reflective metasurface and study these cases individually. First, we assume that the unit cells can alter the phase response of the incoming reference-wave across the full phase-range, $0-2 \pi$. For this scenario, we investigate two different quantization levels, 1-bit and 4-bit. We consider that the reflective metasurface is illuminated by an arbitrarily selected plane-wave incident along optical axis (z-axis) of the surface. The objective function is defined to be a wavefront radiated in the broadside direction $\left(\theta=0^{\circ}, \phi=0^{\circ}\right)$ upon reflection from the surface. The reflective surface patterns shown in Figs. 1(a) and (b) are generated in MATLAB while we make use of a full-wave EM simulation software, CST Microwave Studio, to simulate the radiation pattern of the reflective surface. Fig. 1(c) shows the radiation pattern of the surface vs varying phase quantization levels.

(a)
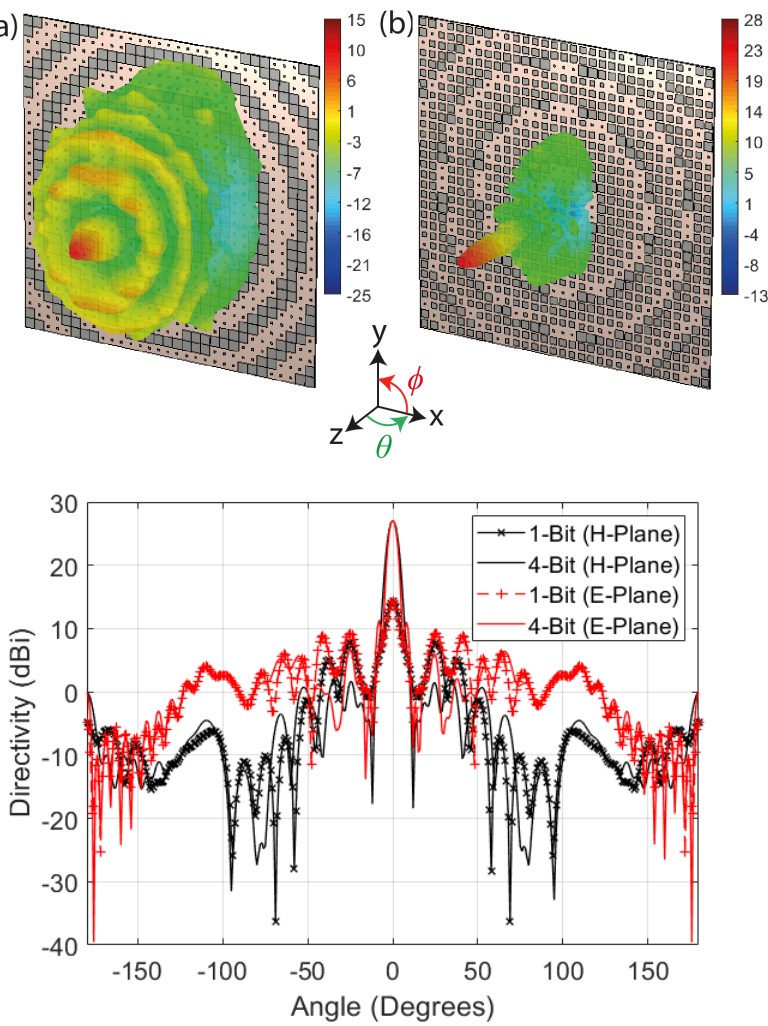

Fig. 1: 3D Radiation pattern of the reflective surface as a function of phase quantization level (a) 1-bit unit cell phase quantization (b) 4-bit unit cell phase quantization (c) comparison of the radiation patterns along the azimuth plane (H-plane) and elevation plane (E-plane). Colorbar in $\mathrm{dB}$ scale. 
In Fig. 1, the H-plane is the azimuth plane (or xz-plane) with $\phi=0^{\circ}$. Similarly, the E-plane is the elevation plane (or yz-plane) with $\phi=90^{\circ}$. Although, from a design perspective, using a 1-bit quantization level would be rather simple, in comparison to the 4-bit quantization case, the radiation pattern exhibits substantially higher sidelobes ( $8 \mathrm{~dB}$ higher) and less directivity $(21 \mathrm{~dB}$ lower in the broadside direction). Increasing sidelobes is a significant concern for wireless communication networks due to the possibility to introduce strong interference across other channels. Similarly, reduced directivity adversely affects the link budget. As a result, from Fig. 1, it is evident that there is a direct relationship between the phase quantization level and the fidelity of the radiation pattern of the reflective surface. We now study the effect of the achievable unit cell phase range on the radiation pattern of the reflective surface. We select the quantization level to be maximum (4-bit) and investigate two unit cell phase ranges, $0-\pi$ and $0-2 \pi$, respectively. The radiation pattern of the reflectarray surface vs the achievable unit cell phase range is shown in Fig. 2.

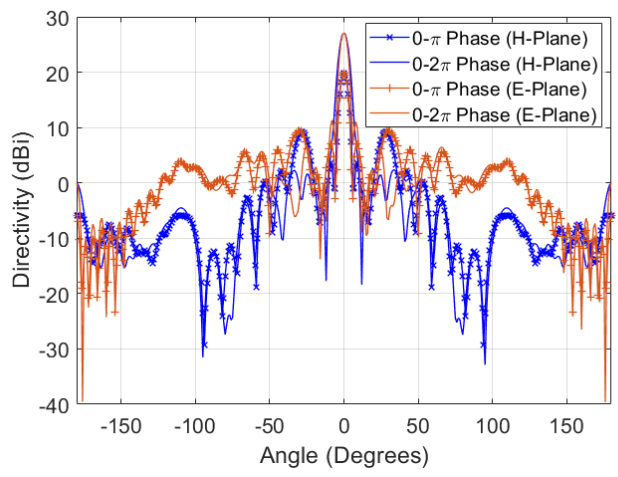

Fig. 2: Radiation pattern of the reflective surface as a function of unit cell phase range.

From Fig. 2, limiting the unit cell phase range to $0-\pi$ reduces the directivity of the surface by $7.3 \mathrm{~dB}$ while increasing the first sidelobe levels by $4 \mathrm{~dB}$ and secondary sidelobe levels by as much as $7 \mathrm{~dB}$. From Figs. 1 and 2, to have a realistic estimate of the link budget in a communication channel and a better understanding of interference characteristics, it is important that the design limitations of reflective surfaces are taken into account. Yet, it is often assumed that the reflective surface is ideal, suggesting that the link budget calculations do not consider the phase quantization errors and assume that unit cells can modulate the phase response of the incident reference-wave across the full-spectrum, 0-2 $\pi$.

Challenge 2: Dynamic reconfigurability and IRS

Albeit producing highly desirable radiation characteristics, conventional reflective surfaces are static, hence, beam characteristics are hard-coded into the surface during the design process. Different from static metasurfaces, an IRS has the capability to dynamically tune the reflection response of the aperture in an all-electronic manner. This is particularly important as communication environments have dynamic characteristics, namely variations in the number of connected users and non-static location distribution over time. Thus, the capability to intelligently change the characteristics of the reflected wavefront to meet their dynamic metrics plays a crucial role in future wireless communication systems. The dynamic modulation of the IRS can be achieved using low-power semiconductor elements, e.g., PIN diodes [6]. This advantage of IRS makes them a promising candidate to replace the power-hungry phase shifting apertures.

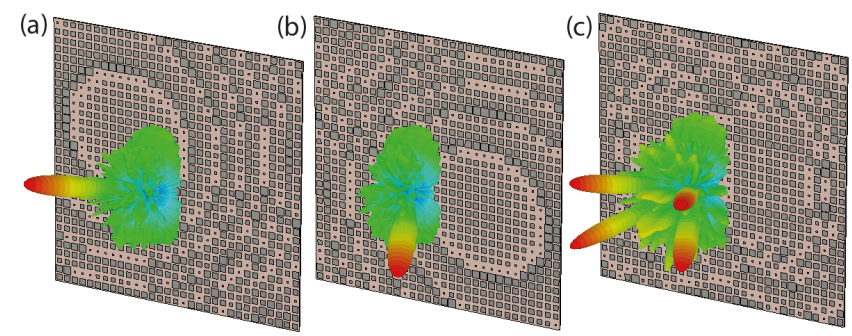

Fig. 3: IRS aperture and electronically steered radiation patterns (a) $\left(\theta=-30^{\circ}, \phi=-30^{\circ}\right)$ (b) $\left(\theta=30^{\circ}, \phi=-\right.$ $\left.30^{\circ}\right)$ (c) multibeam pointing in $\left(\theta= \pm 30^{\circ}, \phi= \pm 30^{\circ}\right)$.

In Fig. 3, we study an IRS that can dynamically control the direction of the reflection. Similar to the unit cell phase studies, for the IRS shown in Fig. 3 the reference-wave is selected to be incident along the optical axis of the reflective surface (z-axis) and it is fixed for all the beam-steering scenarios studied in Fig. 3. Moreover, we consider a 4-bit phase quantization and assume that the unit cells exhibit a phase range of $0-2 \pi$. Once these design challenges are satisfied, IRS can produce reconfigurable radiation patterns to an excellent fidelity, rendering them perfectly suitable for $6 \mathrm{G}$ networks.

\section{CEll-Free massive MIMO}

Cell-free massive MIMO (CF MaMi) has been proposed in [7] to overcome the boundary effect 
of cellular networks. In CF MaMi, many access points (APs) distributed in a geographic coverage area coherently serve many users in the same timefrequency resources. There are no cells, and hence, no boundary effects. Key points of CF MaMi:

- CF MaMi relies on MaMi technology. More precisely, using many APs, CF MaMi offers many degrees of freedom, high multiplexing gain, and high array gain. As a result, it can provide huge energy efficiency and spectral efficiency. These gains can be obtained with simple SP due to the favorable propagation and channel hardening properties of MaMi technology. Note that in CF MaMi, we still have channel hardening, but potentially at a reduced level to that observed in colocated MaMi.

- In CF MaMi, the service antennas (APs) are distributed over the whole network, and hence, we can obtain macro-diversity gains. As a result, CF MaMi can offer a very good network connectivity, i.e., it provides good services for all users in the networks. There is no dead zone. Figures $4 a-4 b$ show the downlink achievable rates displayed with scaled colors for $\mathrm{CF} \mathrm{MaMi}$ and colocated MaMi, respectively. Clearly, CF MaMi can offer much more uniform connectivity for all users.

- Different with colocated MaMi where the base station is equipped with very large antennas, in CF MaMi each AP has a few antennas. Thus, CF MaMi is expected to be built by low-cost, low-power components and simple SP APs.

The above benefits (in particular the high network connectivity) fulfil the main requirements of future wireless networks. Therefore, CF MaMi has become one of the promising technologies of beyond $5 \mathrm{G}$ and towards 6G wireless networks, and attracted a lot of research interest, see [8] and references therein.

Challenge 3: Practical user-centric approaches

In canonical CF MaMi [7], all APs participate in serving all users through the backhaul connections with one or several central processing units (CPUs). This is not scalable in the sense that it is not implementable when the network size (number of APs and/or number of users) grows large. Designing a scalable structure is one of the main challenges of CF MaMi. It is shown in [7] that, owing to the path loss, only $10-20 \%$ of the total number of APs really participate in serving a given user.

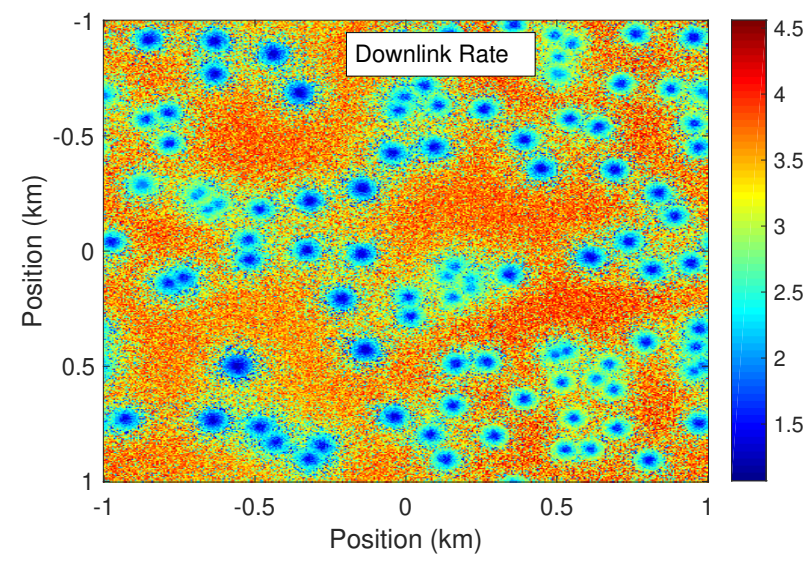

(a) $\mathrm{CF}$ MaMi

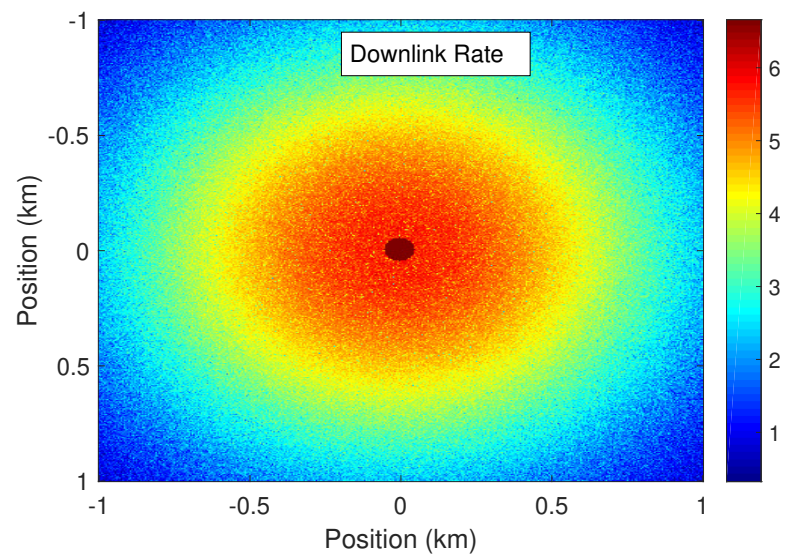

(b) Colocated MaMi

Fig. 4: The downlink achievable rates for different user locations displayed with scaled colors (obtained using simulation approach described in [7]); (a): 100 APs are uniformly located at random in a $2 \mathrm{~km} \times 2 \mathrm{~km}$ area; (b): all 100 service antennas are located at the original point (i.e. the center of the square area).

Thus, each user should be served by a subset (not all) of APs. There are two ways to implement this: network-centric and user-centric approaches. In the network-centric approach, the APs are divided into disjoint clusters. The APs in a cluster coherently serve the users in their joint coverage area. Networkcentric-based systems still have boundaries, and hence, are not suitable for CF MaMi. By contrast, in user-centric approach, each user is served by its selected subset of APs. There are no boundaries, and hence, user-centric approach is a suitable way to implement CF MaMi. There are several simple methods to implement user-centric approach, such as each user chooses some of its closest APs or chooses a subset of APs which contribute most 
of the total received power of the desired signal [9]. Yet, existing methods are not optimal, and still require huge connections from all APs to the CPUs. In addition, the cluster formed by each user changes quickly depending on the user locations. This requires more control signaling. Hence, designing a practical user-centric approach is a challenging exercise and requires urgently intensive research.

\section{Challenge 4: Scalable power control}

Power control is central in $\mathrm{CF}$ MaMi since it controls the near-far effects and the interuser interference to optimize the objectives (e.g. the maxmin fairness or the total energy efficiency) we want to achieve. Ideally, power control is done at the CPU under the assumption that the CPU perfectly knows all large-scale fading coefficients. Then, the optimal power control coefficients will be sent to the APs (for the downlink transmission) and to the users (for the uplink transmission). This requires huge front/back-hauling overhead. Yet, it is very difficult for the CPU to have perfect knowledge of large-scale fading coefficients associated with a potentially unprecedented number of APs and users. Thus, the above power control method is not scalable with the network size. To make the system scalable, power control should be done distributed at the APs with local knowledge of the channel conditions. This is again problematic because it is hard to control the near-far effects and interuser interference without full channel knowledge of all links from all APs and users. Some heuristic power control schemes have been proposed, which, however, are developed based on a specific assumption of the propagation environment, and hence, it is hard to evaluate how well these schemes work in practice. Promising approaches based on machine learning (ML) and deep learning (DL) have recently been proposed in the IoT context [10]. An important question is whether these approaches are also scalable, in order to meet the foreseeable dimensions and decentralization of $\mathrm{CF}$ MaMi networks.

\section{Challenge 5: Advanced distributed $S P$}

One of the ultimate aims of CF MaMi research is designing a SP scheme which offers good performance and can be implemented in a distributed manner. Otherwise, the system is not scalable. This is very challenging. In canonical CF MaMi [7], conjugate beamforming is proposed to use since it can be implemented in distributed manner and performs well. However, compared to other linear processing schemes such as zero-forcing (ZF) and minimum mean-square error (MMSE), the performance of conjugate beamforming is far below. To cover the gap between conjugate beamforming and $\mathrm{ZF} / \mathrm{MMSE}$, we need to have additionally very large number of service antennas. CF MaMi with local zero-forcing scheme was proposed in [11]. However, this scheme requires that each AP has a large number of antennas. It is more challenging for the uplink designs. Currently, there are no distributed SP schemes available for the uplink in CF MaMi. Even with the simple matched filtering, we need to send the (processed) signals from each AP to the CPUs for signal detection.

\section{MOVING TO HIGHER FREQUENCY BANDS}

Future wireless systems for $6 \mathrm{G}$ will potentially rely on: i) Millimetre-wave technologies (30 to 300 $\mathrm{GHz}$ ); ii) $\mathrm{THz}$ technologies ( $300 \mathrm{GHz}$ to $3 \mathrm{THz}$ ); and iii) Free space optics (FSO). For example, utilizing spectrum available from $100 \mathrm{GHz}$ to $1 \mathrm{THz}$, will have the potential to deliver data rates in excess of 10 Gbps [12].

Challenge 6: Packaging/interconnect techniques In addition to providing for a physical enclosure, packaging must provide a reliable interconnection between interior and exterior operating environments. Some of the key driving factors include integration of high-speed semiconductor integrated circuits with advanced antenna systems and integration with optoelectronics. Materials used must be characterized at $\mathrm{THz}$ frequencies as this data is not readily available. At higher frequencies, bond wires cause considerable signal degradation. The effects of bond wires are difficult to characterize for large signal applications, such as power amplifiers and also for phase critical applications, such as beamformers for phased arrays, where these can introduce undesirable side lobe levels. Traditional metallic split-block packages provide excellent performance but are bulky and heavy. Recent activities indicate noticeable progress in interconnect and packaging technologies for $\mathrm{THz}$ applications. Cutting-edge techniques in micro-machining and LTCC technology yield compact and low-cost solutions. Additive manufacturing techniques, such as metal coated 3D printing of plastic devices can realize low-cost, light weight and compact devices.

Challenge 7: Transceiver design

The compact physical size and power efficiency 
requirements become more challenging at mm-wave and $\mathrm{THz}$ frequencies. Hybrid beam forming will be best suited to implement large number of antenna elements along with high efficiency amplifiers. Performance parameters, such as the noise figure, output power and power efficiency degrade significantly for high operating frequencies. Demodulation of higher order modulated signals also becomes more challenging as phase noise increases at higher frequencies. Advanced array SP techniques must complement the transceiver design to address these challenges. Novel techniques, such as spatially oversampled antennas and new phased array architectures can be leveraged to provide a solution to size, weight and power consumption of large mmwave/THz antenna arrays.

Challenge 8: Measurements \& standardization Two popular approaches for phase-sensitive measurements at $\mathrm{THz}$ use either vector network analyzers (VNAs) or time domain spectrometers [13]. In both cases, calibration, verification, and measurement traceability at $\mathrm{THz}$ frequency bands remains a major challenge. For time domain systems, a major challenge is the establishment of standardized measurement and calibration, whereas for VNA systems, solutions are being sought for high precision waveguides and interconnects. Electro-optic sampling (EOS) shows much promise as a complementary approach to $\mathrm{THz}$ measurements, although it is yet to extend the bandwidth to $1.5 \mathrm{THz}$ and to improve resolution. Beyond this, there is also a pressing need for standardization. More precisely, the establishment of a robust framework which enables meteorological traceability to the International System of Units for $\mathrm{THz}$ measurements.

\section{The Role of SP IN THE 6G ERA}

The journey towards $6 \mathrm{G}$ will inevitably be hurdled by significant challenges in the SP arena. Massively populated and decentralized (cell-free) networks, supporting unprecedented Internet of Everything connectivity, will produce high-dimensional and increasingly complex signals, which are subject to increased interference and other impairments (e.g., related to synchronization, temporal correlation etc.) that have so far been largely overlooked. Current SP methods, generally based on low-dimensional signals and classic stationarity assumptions, will indeed need to be rethought. We now discuss two of these methods, namely channel estimation and adaptive filtering, and their associated challenges.

\section{Challenge 9: Channel estimation}

Extensive research in the context of $5 \mathrm{G}$ has been concerned with the need to reduce training overheads in pilot-based channel estimation. While such need will be most critical in 6G networks, viable solutions become extremely challenging due to the massive scale-up and connectivity demands, in particular: (i) supporting high data rates (Gbps) in high-mobility scenarios - a prime concern of operators-will require dealing with much shorter channel coherence times; (ii) ultra-low latency requirements will see transmission intervals substantially shortened, and (iii) the number of parameters to estimate will be massively large as a consequence of the scaling (not only of antennas/access points, but also in the numbers of users/devices). The high-dimensional channels will therefore need to be estimated in severe under-sampling constraints, which might render pilot-based (coherent) estimation approaches unfeasible, particularly under highmobility or low-latency requirements. Blind (noncoherent) estimation approaches-which do not require dedicated pilot signals - stand as promising alternatives and might play a key role. However, these approaches typically require knowledge of the (high-dimensional) received signal covariance matrix [14], which will again need to be acquired from a limited number of samples. To that end, research in the fields of random matrix theory (RMT) and high-dimensional statistics will be relevant; in particular, to develop accurate estimators of large covariance matrices (and their eigen-spectrum) under limited sampling. Other, more recent approaches based on machine learning (ML) might also play a significant role (see [10] and references therein).

\section{Challenge 10: Adaptive filtering}

In beamforming, transmitted signals are dynamically adapted (via digital precoding) to the propagation conditions, effectively mitigating interference and noise. Adaptive beamforming can be seen as a linear filter with a particular design objective, e.g., to maximize the signal-to-noise ratio (SNR). Optimal solutions for the beamformer (and associated receiver filters) require the covariance matrix of the aggregated interference and noise; unknown in practice, this needs to be estimated from observed samples. Current solutions rely on classical estimators, such as the sample covariance matrix 
(SCM), which will return a poor estimate in highdimensional $6 \mathrm{G}$ scenarios, due to:

- Finite sampling (scarcity of samples): While the numbers of antennas and user devices will scale up massively, strict low-latency and highmobility requirements will impose a rather limited number of training (observed) samples.

- Temporal correlation: The ultra-dense and highly decentralized deployments, e.g., with thousands of distributed access points in $\mathrm{CF}$ networks, will be subject to non-perfect synchronization (e.g., between interference and desired signals) which might in turn produce non-stationarity effects.

- Outlying samples: With millions of interconnected devices (from electrical/smart appliances to connected vehicles), it is also reasonable to expect multiple sources of impulsive noise and, in security-sensitive applications/environments, eventual sources of intentional interference (jamming).

Traditional estimators (e.g., SCM) rely on the sufficient availability of samples (the number of samples should be far greater than the number of signals) and on the assumption that these samples are i.i.d. Under the conditions above, however, the mismatch between true and estimated covariance leads to highly inaccurate filters with severe performance losses, in terms of connectivity, reliability, and data rates. A major challenge is then to develop filtering solutions which are robust to the effects of finite-sampling, temporal correlation, and corrupted (outlying) samples. Relevant tools and promising directions can be leveraged from the fields of robust statistics, RMT, and high-dimensional covariance estimation (see, e.g. [15] and references therein).

\section{CONCLUSION}

Although the $6 \mathrm{G}$ era is a decade away, it is extremely timely to understand what are the main challenges for communications engineers that require immediate research attention. This article has identified ten of the most pressing challenges whose investigation will cross-leverage expertise in signal processing, information theory, electromagnetics and physical implementation.

\section{REFERENCES}

[1] J. G. Andrews, et al., "What will 5G be?," IEEE J. Sel. Areas Commun., vol. 32, no. 6, pp. 1065-82, June 2014.
[2] W. Saad, M. Bennis, and M. Chen, "A vision of $6 \mathrm{G}$ wireless systems: Applications, trends, technologies, and open research problems," [Online], arxiv, 2019. https://arxiv.org/abs/1902.10265.

[3] P. Yang et al., "6G wireless communications: Vision and potential techniques," IEEE Netw., vol. 33, no. 4, pp. 70-75, Jul. 2019.

[4] H. T. Chen, A. J. Taylor, and N. Yu, "A review of metasurfaces: Physics and applications," Reports on Progress in Physics, Vol. 79, No. 7, 076401, 2016.

[5] R. J Mailloux, Phased Array Antenna Handbook, Artech house, 2017.

[6] O. Yurduseven et al., "Dynamically reconfigurable holographic metasurface aperture for a Mills-Cross monochromatic microwave camera," Optics Express, vol. 26, no. 5, 2018.

[7] H. Q. Ngo, et al., "Cell-free massive MIMO versus small cells," IEEE Trans. Wireless Commun., vol. 16, no. 3, pp. 1834-1850, Mar. 2017.

[8] G. Interdonato et al., "Ubiquitous cell-free massive MIMO communications," EURASIP J. Wireless Commun. Netw., vol. 2019, no. 1, p. 197, 2019.

[9] H. Q. Ngo et al., "On the total energy efficiency of cell-free massive MIMO," IEEE Trans. Green Commun. Netw., vol. 2, no. 1, pp. 25-39, 2017.

[10] M. Chen et al., "Artificial neural networks-based machine learning for wireless networks: A tutorial," IEEE Commun. Surveys Tuts., vol. 21, no. 4, pp. 3039-3071, 2019.

[11] G. Interdonato et al., "Local partial zero-forcing precoding for cell-free massive MIMO," [Online], arxiv, 2019, https://arxiv.org/abs/1909.01034

[12] C. Han et al., "Terahertz communications (TeraCom): Challenges and impact on 6G wireless systems," [Online], arxiv, 2019, http://arxiv.org/abs/1912.06040.

[13] M. Naftaly et al., "Metrology state-of-the-art and challenges in broadband phase-sensitive terahertz measurements," Proc. IEEE, vol. 105, no. 6, pp. 1151-1165, Jun. 2017.

[14] H. Q. Ngo and E. G. Larsson, "EVD-based channel estimation in multicell multiuser MIMO systems with very large antenna arrays," in Proc. IEEE ICASSP, pp. 3249-3252, 2012.

[15] N. Auguin et al., "Large-dimensional behavior of regularized Maronna's M-estimators of covariance matrices," IEEE Trans. Signal Process., vol. 66, no. 13, pp. 3529-3542, Jul. 2018.

\section{AUTHORS}

Michail Matthaiou is a Reader at Queen's University Belfast (QUB), UK. His research spans signal processing, massive MIMO, and mm-wave systems.

Okan Yurduseven is currently a Senior Lecturer at QUB. His research interests include microwave and $\mathrm{mm}$-wave imaging, MIMO radar, antennas and metamaterials.

Hien Quoc Ngo is a Lecturer at QUB. His main research interests include massive MIMO, cell-free massive MIMO, physical layer security, and cooperative communications.

David Morales-Jimenez is a Lecturer at QUB. His research interests span statistical signal processing, random matrix theory, and high-dimensional statistics.

Simon L. Cotton is a Professor of Wireless Communications at QUB. Among his research interests are propagation measurements and statistical channel modeling.

Vincent F. Fusco is the Chair of High Frequency Electronic Engineering at QUB. His research interests include active antenna front-end techniques and self-tracking antenna arrays. 